\title{
ACTION TOXIQUE DU CUIVRE SUR LES BRANCHIES DE CARPE (CYPRINUS CARPIO)
}

\author{
par R. Labat ${ }^{2}$ J. Pequignot et A. Chateinet.
}

\begin{abstract}
L'action déplétive du cuivre sur les cellules à mucus au niveau des filaments branchiaux chez la Carpe, a été mise en évidence. Cette déplétion est suivie d'une inhibition des cellules mucosécrétrices, levée seulement après un retour aux conditions normales.
\end{abstract}

\section{Toxic action of copper on the gills of carp (Cyprinus carpio).}

Histological changes in Carp gill structure was observed after poisoning by copper salt. Copper induced a mucus cell depletion, followed by a mucosecretion inhibition, only break a return in normal conditions.

Les métaux lourds $(\mathrm{Pb}, \mathrm{Hg}, \mathrm{Cu}$ et $\mathrm{Zn})$ sont présents à l'état de traces dans l'eau. Tous sont toxiques pour la vie animale, à fortes, ou faibles concentrations, et la plupart son létaux à moins d'un ppm. Dans les régions agricoles, les bassins versants reçoivent des quantités importantes de sels de cuivre, et ce dernier est par ailleurs employé pour traiter certaines infections mycosiques et les attaques de protozoaires chez les poissons.

Il est important de déterminer si cette thérapeutique est farorable à l'animal ou si elle ajoute ses méfaits à ceux des parasites. Ordinairement, les effets toxiques des métaux sont rapidement réduits, soit par précipitation avec le carbonate de calcium, soit par des phénomènes de chélation (Doudoroff et Katz, 1953, Spraguc 1969). Fitzgerald (1963) a montré que le sulfate de cuivre additionné d'acide citrique est 500 fois moins toxique pour certains poissons que lorsqu'il est employé seul. Mais il peut y avoir aussi des effets synergiques de deux métaux lorsqu'ils sont associés (Cuivre et Zinc par exemple) (Doudoroff 1952, Sprague 1964, Herbert 1952).

L'action des métaux lourds sur la survie, la reproduction et la croissance des poissons a fait l'objet de plusieurs travaux (Lloyd 1960, Mount et coll. 1969).

Cette note se rapporte aux effets du cuivre sur les branchies de la Carpe.

1. Université Paul-Sabatier, Laboratoire d'Ecophysiologie Animale, 38, ruc des Trente-Six-Ponts, 31 - Toulouse. 


\section{Matériel et technique.}

Nous avons utilisé une cinquantaine de Carpes de 200 à 300 g, dont une vingtaine pour l'histologie. Les sujets en expérience vivent dans une eau d'une dureté moyenne de $12^{\circ}$ polluée artificiellement selon la technique précédemment décrite (Labat et coll. 1972). Les concentrations varient suivan! les expériences de 0,5 à $1,5 \mathrm{ppm}$ (seuil létal) de $\mathrm{Cu}^{++}$, à la température constante de $8{ }^{\circ} \mathrm{C}$. Cette température est celle de nos premières expériences hivernales, nous nous proposons par la suite de la faire varier jusqu'à $25^{\circ} \mathrm{C}$. La durée normale d'une expérience est de 24 heures, pour la plupart des tests biologiques; cependant, pour l'étude his'ologique des branchies, nous avons prolongé l'action du toxique pendant 48 heures, le seuil létal n'étant pas atteint. De même nous avons effectué des retours aux conditions normales sur des poissons exposés au polluant ( 24 ou 48 heures).

Les prélc̀vements de branchies sont effectués sur l'animal vivant, soit après séjour de 24 el 48 heures dans l'eau polluée, soil 12, 24 et 48 heures après le retour aux conditions normales.

\section{Techniques histologiques.}

Les filaments branchiaux de Carpes intoxiquées par une solution à 1 ppm de Cu sont fixés au liquide de Bouin et inclus à la paraffine. Les sections longitudinales de $5 \mu$ d'épaisseur sont colorées au bleu alcian après coloration nucléaire à l'hématoxyline ou au rouge solide. Sur certaines, on effectue la réaction métachromatique de Lison au bleu de Toluidine ( $\mathrm{pH} 5$ et $\mathrm{pH} 3$ ) ou le test au P.A.S. La numération des cellules à mucus ainsi mises en évidence est effectuée pour chaque échantillon, sur 50 longueurs de $0,32 \mathrm{~mm}$ repérées sur un micromètre et réparties au hasard sur plusieurs préparations identiques. Les photographies réalisées avec des grossissements microscopiques de 250 à 400 sont agrandies 4 fois au tirage.

\section{Résultats et discussion.}

Le séjour en eau polluée par le sulfate de cuivre provoque très rapidement au niveau de la branchic de la carpe une diminution du nombre des cellules à mucus (planches et tableau). On note déjà une baisse de 63 pour cent après 24 heures et de 72 pour cent après 48 heures. Chez les sujets soumis à une solution létale de $\mathrm{SO}_{4} \mathrm{Cu}$, le nombre des cellules à mucus est extrêmement faible par rapport au témoin ( -82 pour cent). Cependant, les affinités tinctoriales du mucus ne sont pas modifiées et la réaction métachromatique de Lison a donné des résultats identiques dans toutes les séries. 

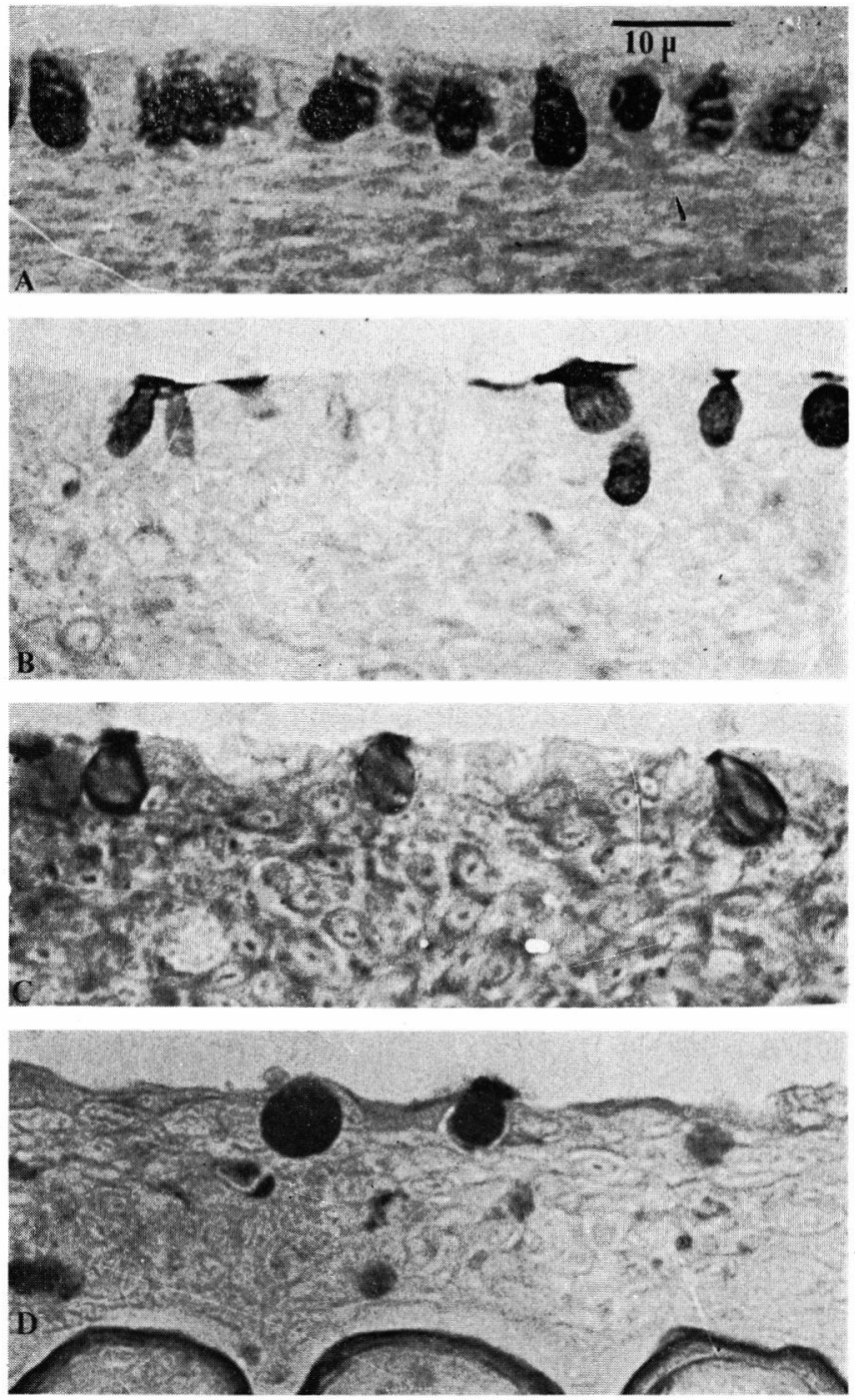

Planche I. - Action du sulfate de cuivre sur les cellules à mucus de la branchie de Carpe. ( $\times 1600$.) Coloration PAS hématoxyline.

I A : Carpe témoin; I B : Après séjour dans sulfate de cuivre $1 \mathrm{ppm} 24 \mathrm{~h}$; I C : Après séjour dans sulfate de cuivre $1 \mathrm{ppm} 48 \mathrm{~h}$; I D : Après séjour dans sulfate de cuivre $1,5 \mathrm{ppm}$ (létal). 

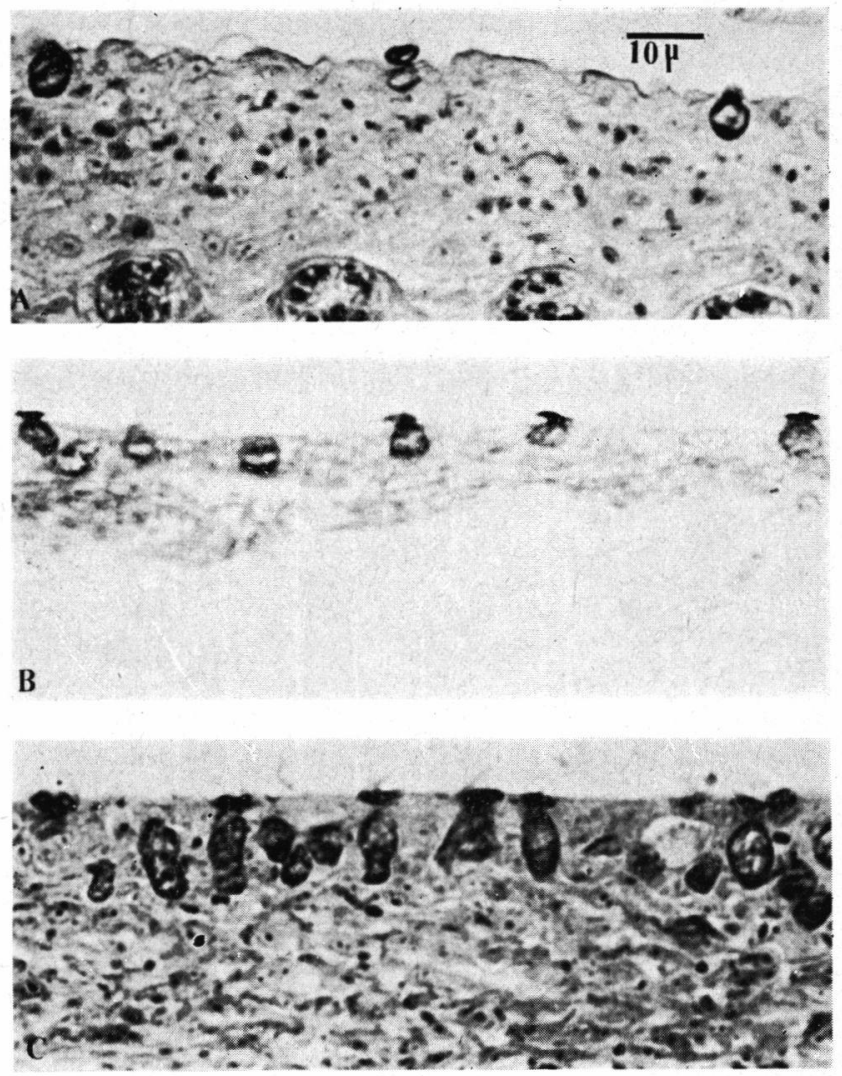

Planche II. - Restauration des cellules à mucus chez des Carpes intoxiquées par du sulfate de cuivre, et après retour aux conditions normales. ( $\times 1000$. II A : Carpe intoxiquée après séjour dans sulfate de cuivre 48 heures; II B : Carpe intoxiquée 48 heures puis retour à la normale 24 heures; II C : Carpe intoxiquée 24 heures puis retour à la normale 24 heures.

Chez les animaux replacés en eau douce courante après leur séjour dans le polluant, l'épithélium excréteur branchial est progressivement restauré. Cependant, la réapparition des cellules ne se fait pas aussi rapidement sur toute la longueur du filament, et l'épithélium branchial a un aspect hétérogène au début de la détoxication. Les cellules à mucus redeviennent colorables et leur nombre est voisin du taux normal chez les animaux dont le séjour en eau toxique n'a pas dépassé 24 heures. Ainsi, il est logique de penser qu'il n'y a pas destruc'ion des cellules à mucus par le sulfate de $\mathrm{Cu}$, mais seulement déplétion. Ceci explique que l'épithélium à mucus redevienne rapidement normal si l'action du 
toxique n'est pas prolongée. Les résultats enregistrés paraissent spécifiques de l'ion Cu et non du sulfate ou de l'abaissement du $\mathrm{pH}$, car des expériences (non publiées) d'intoxication par les ions sulfates sont restées sans effets sur les cellules à mucus.

\section{Conclusion.}

Il nous semble important de souligner l'action déplétive du cuivre sur les cellules à mucus au niveau des filaments branchiaux. Cette déplétion est suivie d'une inhibition des cellules mucosécrétrices levée seulement après un retour aux conditions normales. II est donc permis de penser que l'action du cuivre sur les ectoparasites des poissons est avant tout d'ordre mécanique et constituée par un rejet global et rapide de la couverture muqueuse et des parasites qui s'y trouvent. Il esl non moins certain que si cette action se prolonge, la perméabilité branchiale et la défense générale du poisson doivent être lésées.

Tableav. - Numération des cellules à mucus sur les filaments branchiaux de Carpes intoxiquées par du sulfate de cuivre.

Nombre de cellules $/ 0,32 \mathrm{~mm}$ - Moyenne de 50 mesures $\pm \operatorname{tsm}(t=2)$.

Nombre de cellules à mucus
Différence pour cent par rapport au témoin
Carpe témoin

Sulfate de cuivre $1 \mathrm{ppm}$ pendant 24 heures

Sulfate de cuivre $1 \mathrm{ppm}$ pendant 48 heures

Sulfate de cuivre létal $1,5 \mathrm{ppm}$

$$
\begin{aligned}
& 28,10 \pm 0,62 \\
& 10,41 \pm 0,42 \quad \therefore \quad-62,9 \\
& 7,89 \pm 0,50 \\
& -71,92 \\
& 4,94 \pm 0,40 \\
& -82,27
\end{aligned}
$$

\section{TRAVAUX CITÉS}

Bronie (B. B.) et MaIckel (R. P.). 1962. - Comparative biochemistry of drug metamolism. In pharmacological Meeting, 6 : 299-324.

Dounoroff (P.). 1952. - Some recent developments in the study of toxic industrial wastes. Proc. 4th Pacific North West Indust. Waste Conf. State Coll. of Washington, Pullman, Wash. : 21-25.

DoudorofF (P.) et Katz (M.). 1953, - Critical review of litterature on the toxicity of industrial wastes and their components to fish. II. Metals and salts. Sewage indust. Wastes, 25 : 802-839.

Fitzgerald (G. P.). 1963. - Factors affecting the toxicity of copper to algae and fish. Proc. Amer. Cheur. Soc. Meeting, New York : 21-24. 
Herbert (D. W.). 1952. -- Measurement of the toxicity of substances to Fish. J. Inst. Sew. Purif., $1: 60-68$.

Labat (R.), Dazarola (G.) et Chatelet (A.). 1973. - Principe d'un dispositif technique servant à l'étude des effluents toxiques dans la pollution de l'eau. Bull. Soc. Hist. Nat. Toulouse. (Sous presse.)

Lloyd (R.). 1960. - The toxicity of zinc sulphate to rainbow trout. Ann. Appl. Biol., $48: 84-94$.

Mount (D. I.) et Stephan (C. E.). 1969. - Chronic toxicity of copper to the fathead minnow (P. prometas) in soft water. J. Fish. Res. Bd. Canada, 26 : 2449-2457.

Sprague (J. B.). 1964. - Avoidance of copper-zinc solutions by young salmon in laboratory. J. Water Pollut. Contr. Fed. : 36-990.

Sprague (J. B.). 1969. - Measurement of polluant toxicity to fish. Water Research, 3 et 5 . 